\title{
Étude de la formation de molécules lors de l'ablation laser de graphite en présence d'ammoniac
}

\author{
J. Hermann, C. Vivien, A. Perrone* et C. Boulmer-Leborgne \\ GREMI, UMR 6606, Université d'Orléans, BP. 6759, 45067 Orléans cedex 2, France \\ *Département de Physique, Université de Lecce, CP. 193, 73100 Lecce, Italy
}

\begin{abstract}
Résumé: La synthèse des couches minces par ablation laser réactive nécessite la compréhension des mécanismes d'interaction entre la matière évaporée et le gaz ambiant. Dans ce contexte, nous avons effectué une étude par spectroscopie d'émission du panache plasma formé lors de l'ablation d'une cible de graphite dans une atmosphère d'ammoniac. L'émission spectrale des radicaux formés en phase gazeuse a été observée en faisant varier le délai entre l'impulsion laser et la distance par rapport à la cible. Les spectres expérimentaux de $\mathrm{CN}, \mathrm{CH}$ et $\mathrm{NH}$ ont été confrontés à des spectres théoriques afin de déterminer les températures rotationnelles et vibrationnelles. Ces mesures mettent en évidence l'effet d'échauffement collisionnel du plasma dont le front atteint des températures de l'ordre de $20000 \mathrm{~K}$. De plus, un équilibre collisionnel existe entre les particules lourdes dans le panache. L'influence de la pression du gaz sur la vitesse de la conversion de l'énergie cinétique en énergie thermique et de la thermalisation est montrée.
\end{abstract}

Le procédé de croissance de couches minces par ablation laser réactive fait objet d'étude dans de nombreux laboratoires de recherche. Malgré ces efforts, il existe un manque de connaissance sur la formation des espèces dans le plasma. Récemment, des modèles du type de Monte Carlo ont été employés pour décrire l'expansion du panache d'ablation en présence d'un gaz à basse pression. Cependant, ces modèles sont limités du fait qu'ils ne tiennent pas compte des réactions chimiques en phase gazeuse.

Dans ce contexte, une étude expérimentale par spectroscopie d'émission a été effectuée lors de l'ablation d'une cible de graphite en présence d'ammoniac. Un faisceau laser à excimère ( $\mathrm{KrF} 248 \mathrm{~nm})$ avec une durée d'impulsion de $25 \mathrm{~ns}$ et une énergie de $50 \mathrm{~mJ}$ est focalisé sur une cible de graphite en présence de $\mathrm{NH}_{3}$ à une pression entre 0.05 et 0.5 Torr. La rotation du porte cible empêche de creuser la cible afin de garder les conditions d'interaction laser-surface constantes. L'émission du plasma est
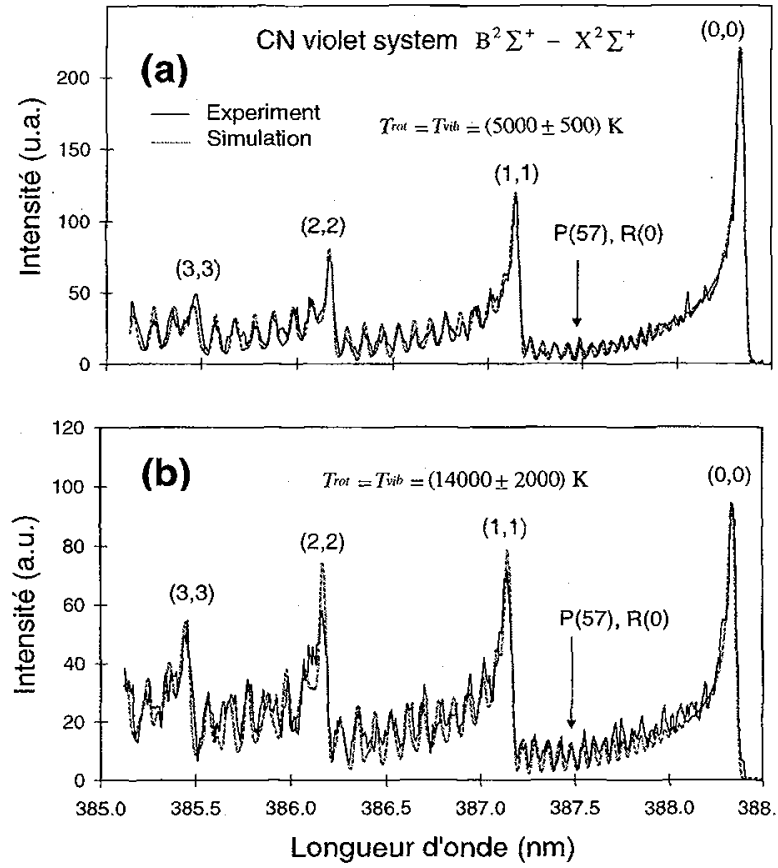
observée perpendiculairement par rapport à l'axe de symétrie du plasma en utilisant deux lentilles qui produisent l'image du plasma dans un plan où est placée l'entrée d'une fibre optique. Un système de translation permet de déplacer l'entrée de la fibre par rapport à l'image et ainsi de varier la distance $z$ de la zone d'observation par rapport à la cible. L'émission est analysée par un spectromètre de haute résolution équipé en sortie d'une matrice $\mathrm{CCD}$ avec une porte de $20 \mathrm{~ns}$ comme détecteur de photons. La fenêtre spectrale est d'environ $4 \mathrm{~nm}$, la résolution spectrale est $1.5 \times 10^{4}$. Pour augmenter le rapport signal-surbruit, l'acquisition des spectres est effectuée en moyennant sur 500 tirs laser. La figure 1 montre deux spectres du système violet $\mathrm{B}^{2} \Sigma^{+}$$\mathrm{X}^{2} \Sigma^{+}$du radical $\mathrm{CN}$ qui ont été enregistrés pour $z=2 \mathrm{~mm}, t=500 \mathrm{~ns}$ (a) et $z=6 \mathrm{~mm}$, $t=300$ ns (b). Il s'agit de la séquence vibrationnelle $\Delta v=0$. Les têtes de bande de $v$ $=0$ à 3 sont identifiées. Par rapport à l'enregistrement présenté dans la figure (a), le spectre dans la figure (b) est caractérisé par 

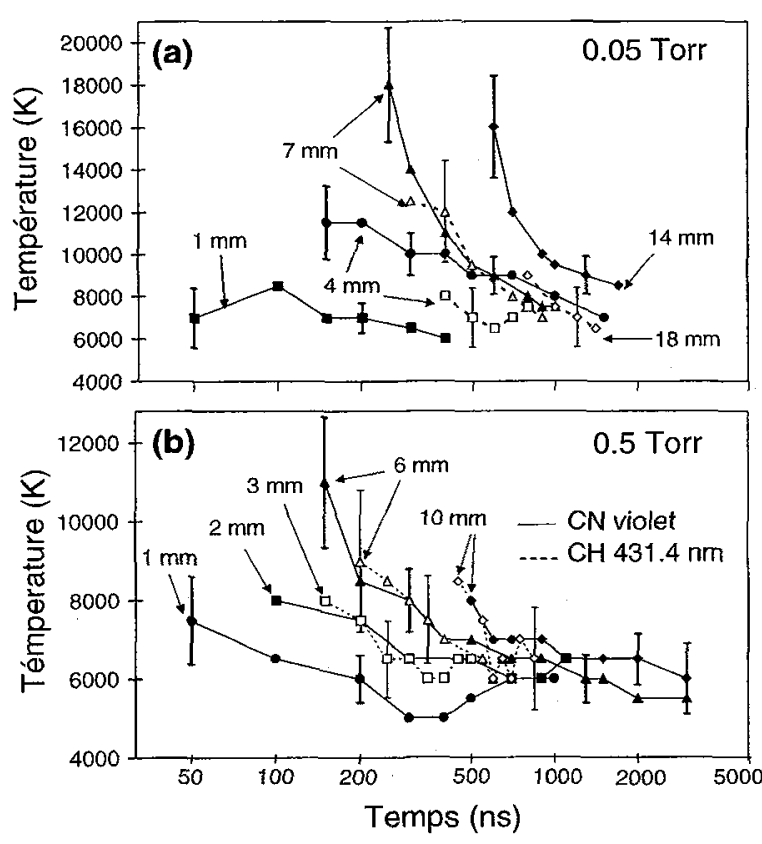

une distribution d'intensité différente. D'une part, la décroissance de l'intensité en fonction du nombre quantique rotationnel est plus rapide pour le spectre (a) indiquant que la température rotationnelle $T_{r o t}$ est plus faible par rapport à celle correspondante au spectre (b). D'autre part, l'intensité relative des têtes de bande $(1,1)$, $(2,2)$ et $(3,3)$ par rapport à celle de la tête de bande $(0,0)$ est plus élevée pour le spectre (b) ce qui prouve que la température vibrationnelle $T_{\text {vib }}$ est supérieure. Pour déterminer $T_{\text {rot }}$, la structure rotationnelle du spectre a été simulée en utilisant les fonctions d'énergie et les forces d'oscillateurs données respectivement par Herzberg [1] et Kovacs [2] en prenant des constantes moléculaires publiées par Luque et Crosley [3]. La température $T_{v i b}$ a été obtenue à partir de la distribution de l'intensité de la séquence vibrationnelle qui a été calculée en utilisant les coefficients d'Einstein donnés par Knowles [4]. De façon générale, le meilleur accord entre spectres expérimentaux et théoriques a été obtenu pour $T_{r o t}=T_{v i b}$ ce qui indique qu'il existe un équilibre collisionnel entre les particules lourdes dans le plasma. On déduit $T_{r o t}=T_{v i b}=5000$ et $14000 \mathrm{~K}$ respectivement pour les figures (a) et (b). Des analyses similaires ont été faites à partir de l'émission des radicaux CH et NH. La figure 2 montre l'évolution spatio-temporelle de la température déduit des spectres du système violet de $\mathrm{CN}$ et du système $431.4 \mathrm{~nm} \mathrm{~A}{ }^{2} \Sigma^{+}-X^{2} \Sigma^{+}$de $\mathrm{CH}$ pour une pression de 0.05 (a) ou 0.5 Torr (b). La figure 2 montre que les températures obtenues à partir des spectres de $\mathrm{CN}$ et de $\mathrm{CH}$ sont identiques dans l'incertitude de mesure ce qui confirme l'hypothèse de l'équilibre collisionnel. Pour une distance d'observation $z$ donnée, la température décroît avec le temps. La valeur initiale, qui correspond au front de la vapeur de carbone en expansion, augmente avec $z$ jusqu'à ce qu'elle atteigne un maximum, puis $T$ diminue. Cette évolution fait preuve d'un effet d'échauffement collisionnel qui résulte de l'interaction entre la vapeur de carbone et le gaz. L'énergie cinétique de la vapeur de carbone est transformée en énergie thermique et énergie interne par collisions entre les particules de la vapeur et celles du gaz. Par rapport à $P_{g u z}=0.05$ Torr (a), la transformation de l'énergie est plus rapide à 0.5 Torr (b) et $T_{\max }$ est atteint à une distance plus faible. Du fait des taux collisionnels supérieurs à 0.5 Torr (b), la thermalisation avec le gaz froid est également plus rapide et $T$ décroît plus rapidement.

Les résultats présentés donnent des informations sur les processus d'interaction entre la vapeur de carbone et l'atmosphère d'azote dans la zone lumineuse limitée à une distance de $z=15 \mathrm{~mm}$. Le substrat étant placé à une distance d'environ $4 \mathrm{~cm}$ où le plasma est peu lumineux, des mesures de fluorescence induite par laser sont envisagées afin d'obtenir des renseignements complémentaires par la détection des particules dans l'état fondamental.

\section{Remerciements}

Ce travail a été soutenu par les gouvernements français et italien dans le cadre d"un programme GALILEE (N097030).

\section{Références}

1. Herzberg G 1950 Molecular Spectra and Molecular Structure: Spectra of Diatomic Molecules 2 nd edn (London: Van Nostrand Reinhold).

2. Kovacs I 1969 Rotational Structure in the Spectra of Diatomic Molecules (Budapest Akademiai Kiado').

3. Luque J and Crosley D R 1998, LIFBASE: Database and Spectral Simulation Program, SRI Internat. Report MP 98-021.

4. Knowles P J, Werner H J, Hay P J and Cartwright D C 1988 J. Chem. Phys. 897334. 